\title{
P 156 DRUGS AT THE END OF LIFE: AN APPROPRIATE CONCERN?
}

Jonathan Pickard, Rosanna Smailes, Maria McKenna. Northern Deanery, Newcastle-upon-Tyne, Great Britain

\subsection{6/bmjspcare-2014-000654.197}

Background The Independent Review of the Liverpool Care Pathway (LCP) described concerns about use of opioid and sedative drugs at the end of life. Baroness Neuberger reiterated the importance of appropriate use of medications to achieve a peaceful death. One concern was that a continuous subcutaneous infusion (CSCI) was started when dying was diagnosed or the LCP commenced, regardless of symptoms.

Aim To investigate the use of medications at the end of life.

Method A retrospective audit of deaths within one hospital was undertaken in July 2013.

Results Of 44 hospital deaths over a one-month period, $82 \%$ $(n=36)$ were deemed expected. 56\% (20/36 patients) were prescribed anticipatory medications for symptoms of pain, agitation, nausea, vomiting and secretions by the appropriate route and frequency. $100 \%$ of anticipatory PRN prescriptions were correctly dosed and $83 \%$ were appropriately prescribed parenterally.

$72 \%$ of patients utilised anticipatory as required (PRN) medication. A CSCI was used in 42\% (15/36) of cases. They most commonly contained an opioid to manage pain (93\%) and/or a benzodiazepine for agitation (73\%), but also included anti-emetic (20\%) and anti-secretory drugs (13\%). The rationale for CSCI commencement was clearly apparent in $7 / 15$ cases; most commonly it was due to frequent use of PRN drugs $(n=6)$ or as a switch from a regular oral opioid $(n=1)$. A CSCI was used for a mean of 3.9 days.

One limitation of these findings is the reliance on written documentation within the patients' medical notes.

Conclusion and Recommendations This audit demonstrated that the majority of patients do not receive medication via a CSCI at the end of life, but that most are administered PRN medication for their comfort. The rationale for starting a CSCI should be clearly documented, specifically recording indication for use and communication with patient, family and carers. 